\title{
Efficient Planar-Integrated Free-Space Optical Interconnects Fabricated by a Combination of Binary and Analog Lithography
}

\author{
Richard Heming, Lars-Christian Wittig, Peter Dannberg, Jürgen Jahns, Ernst-Bernhard Kley, and Matthias Gruber
}

\begin{abstract}
Design, fabrication, and experimental testing of an integrated microoptical module for interconnection are reported. The systems integration is based on the concept of planar-integrated free-space optics. The module combines diffractive-reflective and refractive microoptics. The diffractive elements were fabricated by binary lithography and reactive ion etching. The refractive elements were made by analog lithography using a high-energy beam sensitive mask and replication in Ormocer. The fabricated module implemented a simple one-dimensional optical interconnect. Two versions were implemented for which insertion losses of approximately 8 and $4.5 \mathrm{~dB}$ were measured, respectively.
\end{abstract}

Index Terms-Analog lithography, microoptics, optical interconnects, planar integration, replication.

\section{INTRODUCTION}

$\mathbf{O}$ PTICAL technologies are believed to play an increasingly important role in many information-technology and sensing applications. One particular field in which optics offers substantial performance improvements is (short-haul) data communication [1]. Optical interconnects are considered as a potential solution for the aggravating communication bottleneck. There have been various approaches to combine optical fibers, optical waveguides, free-space optics, and optoelectronic emitters and detectors into common technological platforms [2], [3]. For the realization of rugged and low-cost devices suitable microintegration and packaging concepts play an important role. Planar-integrated free-space optics (PIFSO) [4] has been demonstrated as a viable approach to build compact and highly functional microoptical systems, especially for interconnect applications [5]-[8].

A PIFSO system integrates all free-space optical components on the surfaces of a transparent substrate several millimeters thick. Typically, most of these components are reflective such that light signals propagate largely inside the substrate on zigzag paths along a tilted and folded optical axis. Since the optical elements are located on the substrate surfaces, they can be fabri-

Manuscript received July 10, 2007; revised January 4, 2008. Published August 29, 2008 (projected).

R. Heming, J. Jahns, and M. Gruber are with the Fakultät für Mathematik und Informatik, Fern Universität in Hagen, 58084 Hagen, Germany (e-mail: matthias.gruber@fernuni-hagen.de).

L.-C. Wittig anf E.-B. Kley are with the Institut für Angewandte Physik, Friedrich-Schiller-Universität Jena, 07743 Jena, Germany.

P. Dannberg is with the Fraunhofer Institut für Angewandte Optik und Feinmechanik IOF, 07745 Jena, Germany.

Color versions of one or more of the figures in this paper are available online at http://ieeexplore.iee.org.

Digital Object Identifier 10.1109/JLT.2008.919456 cated with high precision using lithographic techniques, micromachining, and/or replication. Using the flexibility of microoptical design and fabrication, a variety of signal-processing tasks can be realized such as optical imaging, routing, beam splitting and fanout, and optical correlation. Especially diffractive optical elements (DOEs) can implement almost any required optical functionalty. However, DOEs fabricated as multilevel structures by binary lithography are, in general, not $100 \%$ efficient. In a PIFSO interconnect with several DOEs and surface reflections in series, losses can easily accumulate to $10 \mathrm{~dB}$ or more [7].

In order to build highly efficient PIFSO systems, advanced fabrication techniques have to be considered. This is possible, for example, by combination of refractive and diffractive microoptics. In their properties, these may almost be viewed as complementary. Refractive elements usually allow high efficiency, but their functionality is limited to prisms and lenses. By contrast, diffractive elements allow high design flexibility, but their efficiency may sometimes be low. This suggests the following design approach: use refractive elements for simple functionality but high efficiency (e.g., beam deflection) and use diffractive optics where specific functionality is required (e.g., beam splitting, wavefront correction).

In earlier work, we investigated the use of analog HEBSglass-based lithography for realizing prisms used as coupling elements [9]. Here, we extend this approach to the realization of a complete integrated PIFSO module that implements one-to-one interconnects between two mechanically transferable (MT)-fiber connectors with high efficiency. In particular, we demonstrate the combined use of binary and analog lithography for PIFSO. In order to further show that the fabrication concept can be implemented at low cost, part of the optics was replicated in a polymer (Ormocer). In the following, we will describe the systems design, fabrication, and testing.

\section{Systems DESIGN}

There are two main reasons to select fiber-to-free-space-tofiber coupling between MT-connectors as the functionality to be implemented in the experimental demonstrator. First, since fiber-optic communication has become the standard in the longdistance domain and since parallel free-space optical interconnects are gaining ground for short-range communication, there is a general need for suitable parallel coupling mechanisms between guided-wave and free-space optical communication hardware. Secondly, the envisaged system architecture makes a realistic comparison between different technological approaches possible because PIFSO modules with the same functionality 


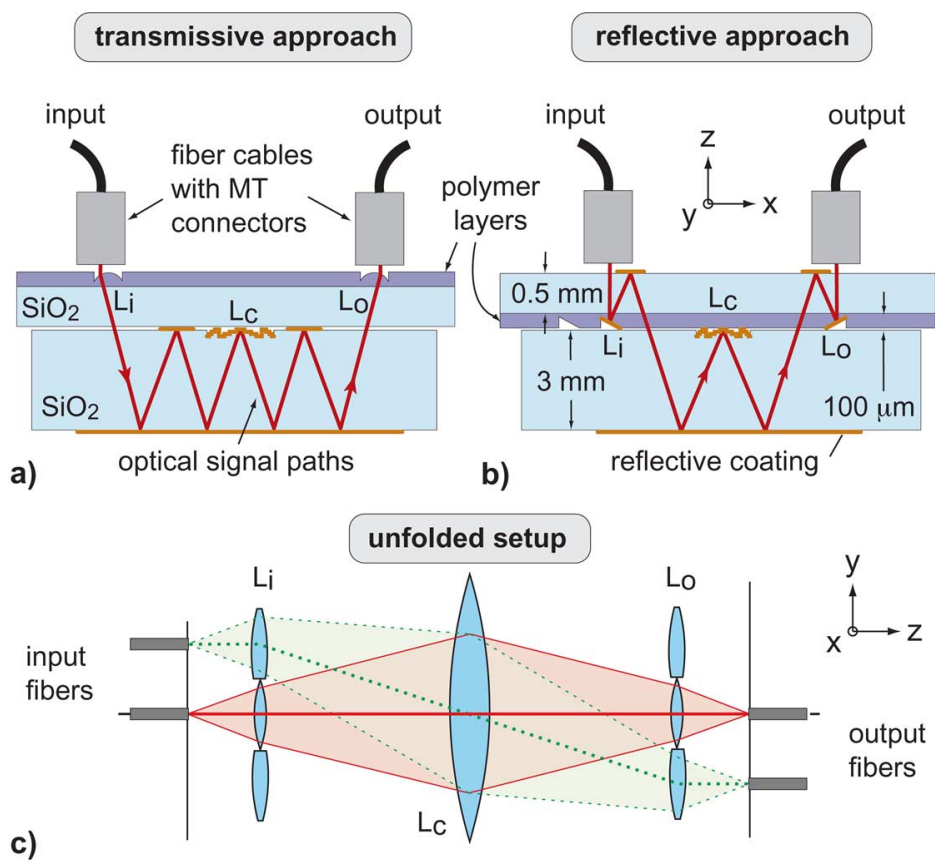

Fig. 1. (a) Transmissive and (b) reflective systems approach for the diffractive-refractive PIFSO interconnect module. (c) Equivalent unfolded version of both approaches.

TABLE I

List OF IMPORTANT GEOMETRICAL DESIGN PARAMETERS

\begin{tabular}{|l|c|}
\hline \multicolumn{1}{|c|}{ design parameter } & value \\
\hline thickness of upper substrat & $0.5 \mathrm{~mm}$ \\
\hline thickness of lower substrat & $3 \mathrm{~mm}$ \\
\hline thickness of polymer layer & $100 \mu \mathrm{m}$ \\
\hline maximum possible sag for elements in polymer & $40 \mu \mathrm{m}$ \\
\hline x-separation of MT-connectors (reflective approach) & $4.4 \mathrm{~mm}$ \\
\hline X-separation of MT-connectors (transmissive approach) & $5.6 \mathrm{~mm}$ \\
\hline N.A. of imaging systems & 0.25 \\
\hline
\end{tabular}

have been realized before using all-diffractive technology [5], [7]. Their optical performance constitutes the benchmark that we aim to improve with the new combined diffractive-refractive systems approach. With comparability as the top priority, we accept that simple one-to-one interconnects do by far not exploit the full topological interconnect potential of PIFSO and can be realized efficiently by alternative technological means.

The idea is to fabricate the thin diffractive components by means of binary lithography and reactive ion etching, and the thick refractive or reflective components by means of analog lithography plus replication into a thin polymer layer. Although both types of components can be integrated monolithically in principle, we decided to use separate substrates for the two technologies here to be able to split the work and to reduce the risk of defects. Our two basic architectural approaches are shown in Fig. 1(a) and (b). Both are fundamentally equivalent, which becomes obvious when they are depicted in unfolded form [Fig. 1(c)]. The distinction lies entirely in the top substrate, which contains the thick optical components. They are used in transmission in one approach and in reflection in the other. Signal paths are somewhat more complex in the latter case; however, reflective optical components require a considerably (typically six times) smaller sag for the same optical functionality than refractive components, which can be a crucial advantage in terms of manufacturability.

The free-space interconnection between the two fiber cables is established through an optical imaging of the end faces of the two MT-connectors onto each other. The focusing power for this operation is mainly provided by the central lens $L_{c}$, which is located on the lower substrate and acts globally for all 12 channels of the connector. By contrast, the in- and outcoupling is carried out individually for each channel by the microlens arrays $L_{i}$ and $L_{o}$. Refering to the two system approaches of Fig. 1, these microlenses are designed as refractive and reflective elements, respectively, and located on the upper substrate. They contribute part of the focusing power for the input-output imaging operation. Their main function, however, is that of a field lens, i.e., they deflect the incoming beams towards the center of $L_{c}$ and the outgoing ones along the axes of the fibers in the MT-connector. As shown in Fig. 1, there are essentially four zigzag propagation steps of optical signals within the PIFSO part in both system versions.

The fundamental geometrical parameters for the above optical system designs are listed in Table I. It was a design goal 


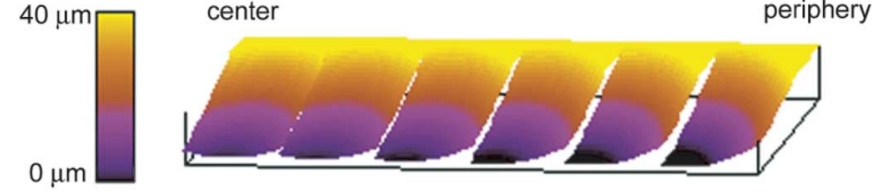

Fig. 2. Calculated height profiles of six of the 12 coupling lenses of arrays $L_{i}$ and $L_{o}$ for the reflective systems approach.

that the imaging systems be able to accommodate multimode optical fibers as input sources. Hence, the numerical aperture (NA) had to be at least as large as that of multimode fibers (in our case NA $=0.25$ ) without leading to overlaps of the folded beams at the substrate surfaces. With the geometry parameters of Table I, a simple paraxial scalar treatment of the imaging operations is possible, polarization effects can be neglected, and a sophisticated aberration correction is unnecessary. We did, however, perform an elliptical distortion of the central lens $L_{c}$ to compensate astigmatism in first order approximation [8].

It is worth noting that the geometry of the system is strongly determined by technological constraints of the analog lithography process. These are a maximum possible sag of approximately $40 \mu \mathrm{m}$ and the fact that steep profile gradients or even profile jumps cannot be fabricated. One should therefore avoid a collapsing of thicker desired profiles into Fresnel-lens-like truncated profiles; otherwise the imperfect edges would generate a lot of stray light and reduce the energetic efficiency significantly. Furthermore, refractive/reflective components should be located close to focal regions (in Fig. 1 object and image plane) where significantly smaller sags are sufficient to implement a certain desired optical functionality. The designs of Fig. 1 adhere to both of these guidelines, as can also be seen from Fig. 2, which depicts the calculated height profiles of one-half of the in-coupling microlens array for the reflective systems approach. As expected, the more peripheral these microlenses are located in the array, they more off-axis they are operated.

\section{FABRICATION}

As indicated, a combination of binary and analog lithographybased techniques is applied to the fabrication of the experimental demonstrators. Extended information about the (standard) binary techniques may be found in [8] and [9]; here we point out only a few selected details. The central diffractive lens $L_{c}$ comprises eight equidistant phase levels that are realized in three successive lithography and dry-etching steps. The reflective coating consists of Ag with a protective overcoating of Al. The two metals are applied by lithography, thermal evaporation, and liftoff.

\section{A. Gray-Scale Lithography}

Gray-scale lithography has been proven to be a suitable technology for the fabrication of arbitrary three-dimensional surface profiles [10]-[12]. This technology uses the high energy beam sensitive (HEBS) (Canyon Materials, Inc.) as a real grayscale mask in conventional photolithographic contact printing. The HEBS glass mask spatially modulates the exposure intensity and thereby the exposure dose for the photoresist. As the basic principle of analog lithography, this modulation of dosage leads to a modulation of solubility of the resist and will be translated into a continuous surface profile during development.
The grayscale mask was generated by e-beam exposure of a HEBS glass mask blank. For this purpose, we used the variable-shape-beam e-beam writer ZBA 23H (Leica Microsystems, VISTEC) at the Institute of Applied Physics in Jena, Germany. The contact printing was carried out at the i-line with the Resist AZ 4562 (Clariant). The most critical part of this work was the preparation of resist layers with a thickness of about $50 \mu \mathrm{m}$ that have reproducible properties in the lithographic process. This was achieved by using a double layer of resist, an experimentally optimized temperature regime for the softbake, and a sufficient storage time afterwards to allow $\mathrm{H}_{2} \mathrm{O}$ molecules to rediffuse into the resist. This is essential for the photoreaction to take place and is an issue only for thick resist layers because of the low diffusion constant.

To calibrate the dependence of the profile height after development on the electron dosage used to expose the HEBS glass mask, a special prism-like test structure was used (Fig. 3). In two iteration steps, the calibration was improved until the test wedge was measured to have a linear profile. The calibration over both lithographic processes (e-beam exposure of mask and exposure/development of resist) provides higher precision for the fabrication of surface profiles than separate calibrations of these processing steps. As a result, the lenses have a deviation from the desired profile of less than $60 \mathrm{~nm}$ within the working area, which is only $0.2 \%$ of the overall profile height.

One drawback of the applied grayscale lithography is the limited resolution, which depends on the used range of electron dosage for the HEBS-glass exposure. As a rule of thumb, surface inclinations of more than $45^{\circ}$ cannot be realized. This leads to a smoothing of the desired surface profiles and thus to large figure errors at sharp edges. As a consequence, in our application, the usable area of the lenses is lower than its geometrical extension. This fact had been taken into account in the system design. Another issue is the surface roughness of the resist profiles because this leads to stray light within the system. On the one hand, this lowers the coupling efficiency because of energy losses; on the other hand, crosstalk between different signal channels will take place. It turned out that the surface roughness of resist profiles after grayscale lithography was about $30 \mathrm{~nm}$ (rms), and even less after replication of the profiles into Ormocer. The character of the roughness was purely statistical; periodic structures were avoided by an adapted data generation for the electron beam exposure of the HEBS-glass mask. Thus, generation of stray light is minimized.

\section{B. Replication}

Replication can be a precise as well as a cost-effective way to generate microoptical elements [13], [14]. In this investigation, we used an ultraviolet (UV) molding technique [15] because of the following advantages. In the UV molding process, a thin polymer layer (with thickness of 10-100 $\mu$ m depending on structure height) is generated on top of a highly transparent, optically homogeneous, precise, and mechanically stable glass substrate. The thermal expansion of the system is dominated by the glass substrate. As a result, we achieve a high stability and high lateral accuracy of the replicated microoptical elements, which is crucial for the PIFSO approach. From previous investigations, it is known that relative deviations of the lateral position are below 


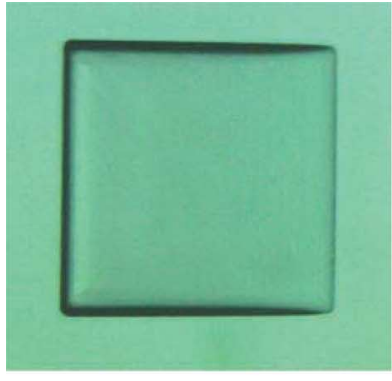

a)

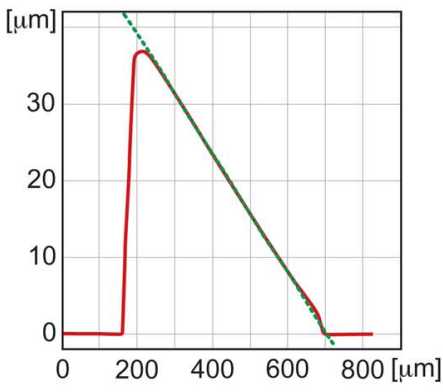

b)

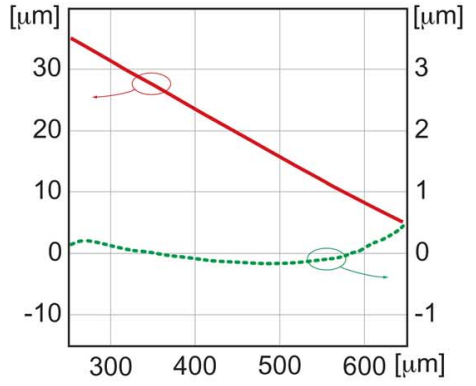

c)

Fig. 3. (a) Linear test wedge used for a quatitative evaluation of the analog fabrication process, (b) theoretical (dashed line) and actual (solid line) profile of the wedge, and (c) central part of the actual profile (solid line) and difference between actual and theoretical curve (dashed line).

$3 \cdot 10^{-5}[15]$ allowing for a proper matching of the pitch of larger arrays. Furthermore, the technology allows for the combination of replicated elements with structures in or on top of the glass substrate. Double-sided replication is possible as well. In addition, the UV-cured polymer shows a higher stability compared to thermoplastic polymers, which is beneficial for the compatibility with further technological steps like metallization or coating with dielectric thin-films, sawing, soldering, or other packaging steps as well as for the long-term stability of the system.

In our investigations, we used a modified mask aligner (SUSS microtec MA6) equipped with a top- and backside alignment microscope for the UV replication. Here the aligner is used for loading the glass substrate as well as the replication tool, for wedge error compensation, setting of the exposure gap (which equals the polymer layer thickness), alignment, and UV exposure. As the UV curing resin, we dispensed an acrylate-type inorganic organic hybrid polymer (ORMOCOMP/ Microresist Technology) that combines high transparency, matched refractive index, low shrinkage, and high thermal and moisture stability. After UV curing and separation from the tool, the replicated element was hard baked at $160{ }^{\circ} \mathrm{C}$ for 30 min under protective gas for optimum stability. Using this technique, upper glass substrates with refractive as well as reflecting elements in a $100-\mu$ m-thick polymer layer were generated. In a future approach, all microoptical elements will be fabricated by doublesided UV molding and subsequent coating.

The finished upper and lower substrates were first inspected and characterized. As an example, Fig. 4(a) shows charge-coupled device (CCD) images of the lower substrate of the reflective demonstrator module; in Fig. 4(b), a scanning electron microscope (SEM) image and a gray-level representation of a whitelight interferogram of polymer lenses from the upper substrate of the same demonstrator are depicted. Apparently there is a good visual agreement with the theoretical profiles shown in Fig. 2. After the characterization, the polymer lens arrays $L_{i}$ and $L_{o}$ of the reflective systems demonstrator were made reflective by evaporating $\mathrm{Al}$ on them with the same technique as used for diffractive components on the lower substrate.

\section{EXPERIMENTS}

A quantitative evaluation of the polymer profiles was carried out with the help of a linear test wedge that was additionally integrated into the replicated system part. Its profile was measured

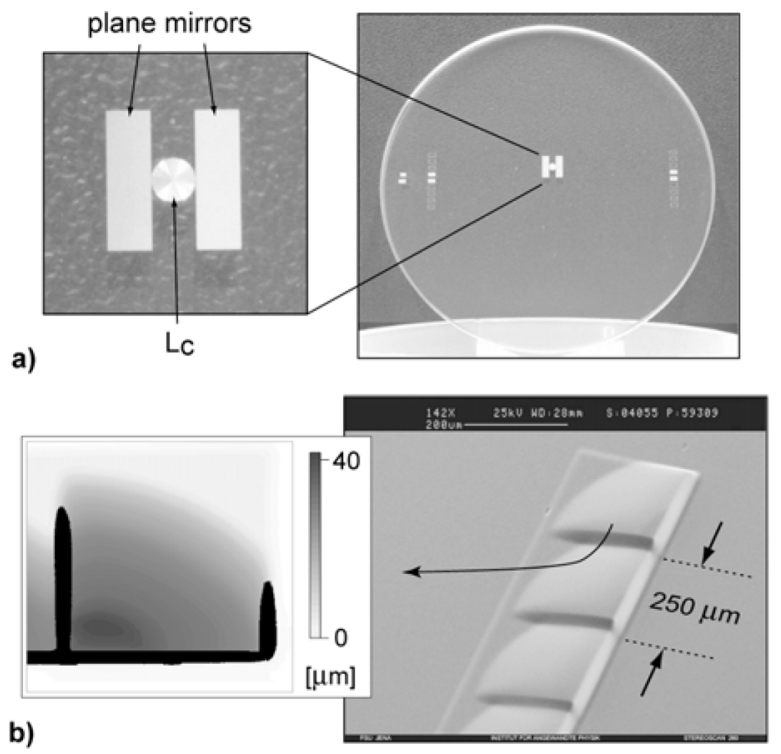

Fig. 4. (a) CCD images of the lower substrate of the reflective systems demonstrator module. (b) White-light interferogram and SEM image of polymer lenses of the upper substrate of the same demonstrator.

with a mechanical profilometer (see Fig. 3). Deviations turned out to be less than $60 \mathrm{~nm}$ at a maximum profile depth of $40 \mu \mathrm{m}$. The profiles of the coupling lenses of both system types were also evaluated [see Fig. 4(b)] and found to have the same level of shape fidelity.

For a qualitative evaluation of the imaging operation between the input and the output MT connectors, the plane in which the front face of the output MT connector would normally lie was observed with a CCD camera. We used a setup as depicted schematically in Fig. 5(a) for the reflective system demonstrator. The experiment was carried out with both single-mode (SM) and multimode (MM) fiber arrays as input sources and a wavelength of $\lambda=850 \mathrm{~nm}$. Fig. 6 shows two representative results when one of the 12 fibers of the input array was illuminated. The two intensity plots at the top thereby correspond to the cross-sections indicated with horizontal dashed lines in the grayscale CCD images at the bottom. The vertical dashed lines mark the nominal center positions of the output fibers and the highlighted sections the diameter of $62.5 \mu \mathrm{m}$ multimode output fibers. 

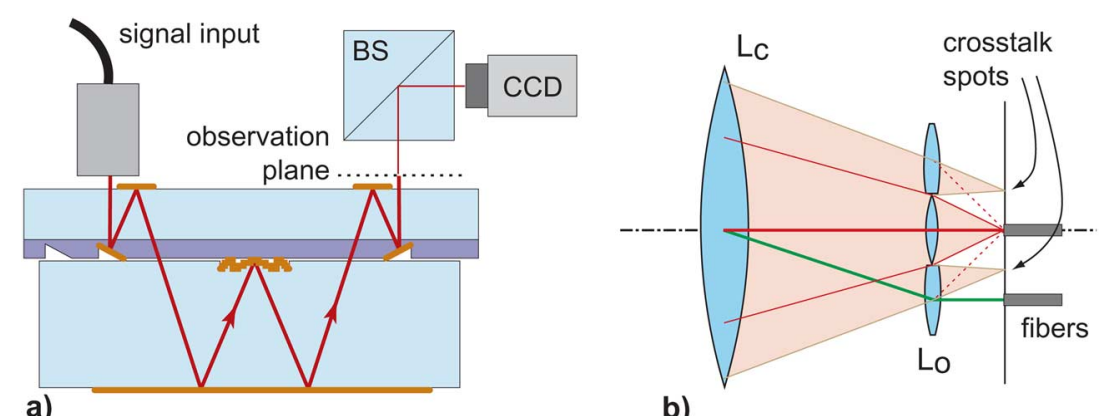

Fig. 5. (a) Qualitative evaluation of the imaging operation with a CCD camera. (b) Geometrical-optical model for the occurrence of crosstalk spots in the observation plane.

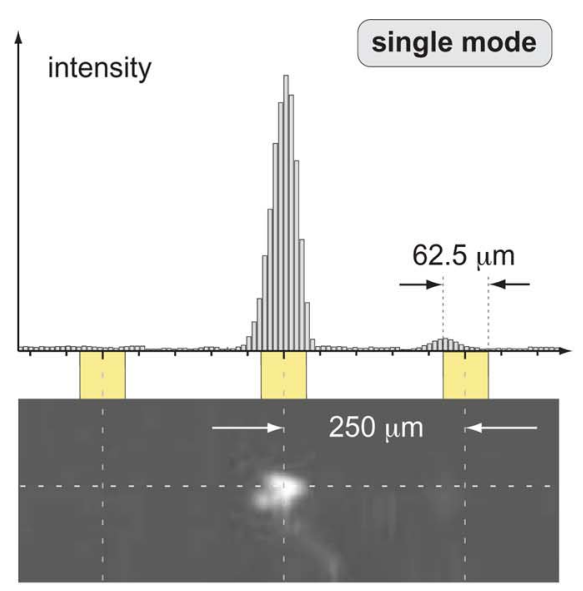

$\operatorname{ch} \# 3$ ch \#2 ch \#1

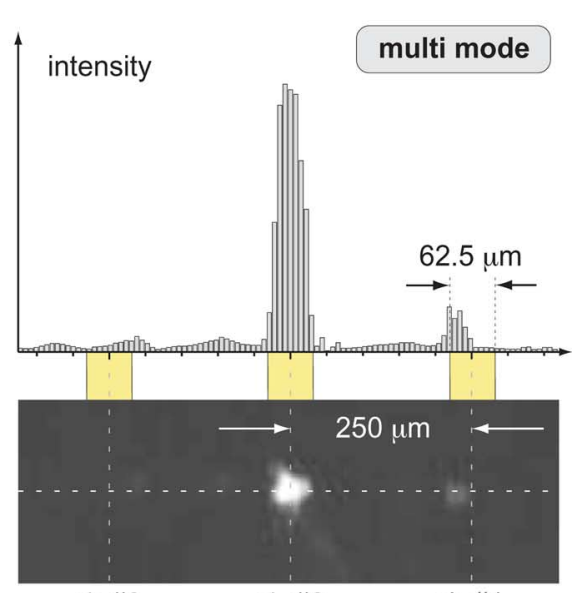

ch \#3

ch \#1

Fig. 6. CCD images of the experiment of Fig. 5(a). The intensity plots at the top correspond to the cross-sections indicated with horizontal dashed lines at the bottom. The crosstalk spots are not coupled into the output fibers due to a lateral deviation of about $30 \mu \mathrm{m}$ from the centers of ch\#1 and ch\#3.

The image spots in Fig. 6 are generated at the correct positions, although the imaging operation is apparently not diffraction-limited. Nevertheless, the spot diameters are small enough that most of the intensity would hit the core area of a $62.5 \mu \mathrm{m}$ MM fiber. The most noticeable (and expectable) difference between SM and MM operation is the larger crosstalk spots in the latter case. As shown in Fig. 5(b), they can occur if the beam diameter at lens $L_{c}$ is somewhat larger than calculated in the paraxial design model due to optical aberrations or misalignments. Then some intensity will fall on the neighboring microlenses in the array $L_{o}$ and generate these satellite spots. Note that the observed crosstalk phenomenon is far less critical than it looks because the satellite spots do not hit the MT connector at the right position nor at the correct angle to be (efficiently) coupled into an output fiber. Hence, they do not necessarily imply a high crosstalk level of the fiber-to-fiber coupling.

For a quantitative evaluation of this fiber-to-fiber crosstalk and the coupling efficiency, the laboratory setup of Fig. 7 was used. It shows the PIFSO module and the two MT connectors mounted on micropositioning stages and perfectly adjusted. Both experimental demonstrators were evaluated with different combinations of SM and MM fibers at the input and the output. Table II lists average values of the measured results. Unfortunately, no measurements could be made with MM input fibers into the transmissive system because the polymer lenses were accidentally destroyed during the mechanical adjustment procedure for this experiment.

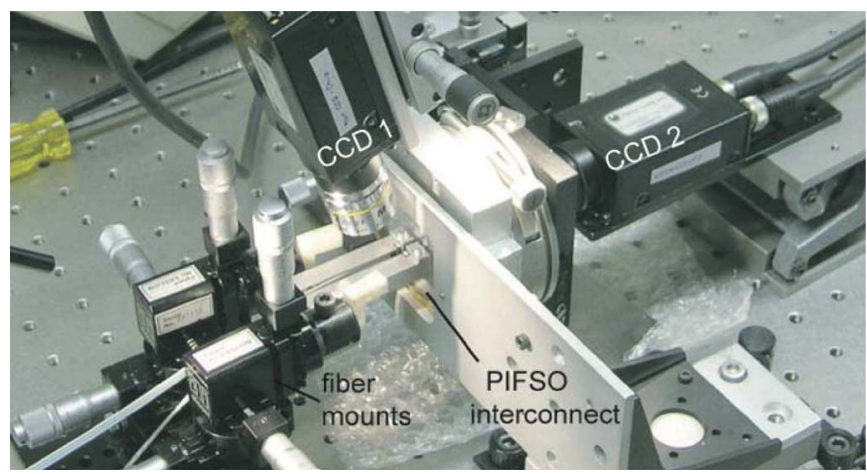

Fig. 7. Laboratory setup for measuring coupling efficiency and crosstalk of the interconnect modules.

Although a theoretical performance estimation of both system approaches resulted in a comparable coupling efficiency, it turns out somewhat unexpectedly that the experimental SM $\rightarrow$ MM coupling efficiency was significantly better with the transmissive systems approach. We believe that this has to do with the last fabrication step in the reflective systems approach, i.e., the thermal evaporation of $\mathrm{Al}$ on the polymer lenses. This process, which is the only substantial fabrication difference of both system approaches, may have caused a slight thermally induced deformation of the polymer lens profiles or a roughening of their surfaces. 
TABLE II

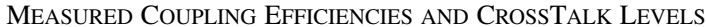

\begin{tabular}{|l|c|c|c|c|}
\hline \multirow{2}{*}{} & \multicolumn{2}{|c|}{ transmissive system } & \multicolumn{2}{c|}{ reflective system } \\
\cline { 2 - 5 } & $\mathrm{SM} \rightarrow \mathrm{MM}$ & $\mathrm{MM} \rightarrow \mathbf{M M}$ & $\mathrm{SM} \rightarrow \mathbf{M M}$ & $\mathrm{MM} \rightarrow \mathbf{M M}$ \\
\hline coupling efficiency & $-4.5 \mathrm{~dB}$ & n.a. & $-8 \mathrm{~dB}$ & $-11 \mathrm{~dB}$ \\
\hline cross-talk & $-43 \mathrm{~dB}$ & n.a. & $-30 \mathrm{~dB}$ & $-24 \mathrm{~dB}$ \\
\hline
\end{tabular}

\section{CONClusion}

We have shown that a PIFSO design approach, according to which refractive elements are used for simple optical functionalities but high efficiency and DOEs for specific complex functionalities, is well suited to combine the advantages of both component types and to obtain energetically efficient PIFSO systems. We have applied this design approach for MT-based fiber-to-PIFSO-to-fiber interconnect modules and demonstrated a fabrication procedure that involves binary and analog lithography as well as replication into Ormocer. In terms of the coupling efficiency, the experimental demonstrators showed an improvement of a factor of three compared to previous PIFSO modules with the same functionality.

\section{REFERENCES}

[1] D. A. B. Miller, "Physical reasons for optical interconnection," Int. J. Optoelectron., vol. 11, pp. 155-168, 1997.

[2] "Special issue on optical interconnections for digital systems," Proc. IEEE, vol. 88, 2000.

[3] R. Baets and L. Vanwassenhove, "2D inter-chip optical interconnect," Opt. Mater., vol. 17, pp. 227-233, 2001.

[4] J. Jahns and A. Huang, "Planar integration of free-space optical components," Appl. Opt., vol. 28, pp. 1602-1605, 1989.

[5] M. Gruber, E. ElJoudi, S. Sinzinger, and J. Jahns, "Practical realization of massively parallel fiber-free-space optical interconnects," Appl. Opt., vol. 40, pp. 2902-2908, 2001.

[6] M. Gruber, "Multi-chip module with planar-integrated free-space optical vector-matrix-type interconnects," Appl. Opt., vol. 43, pp. 463-470, 2004.

[7] M. Gruber, R. Kerssenfischer, and J. Jahns, "Planar-integrated freespace optical fan-out module for MT-connected fiber-ribbons," $J$. Lightw. Technol., vol. 22, pp. 2218-2222, 2004.

[8] M. Gruber and J. Jahns, "Planar-integrated free-space optics-From components to systems," in Microoptics-From Technology to Applications, J. Jahns and K. Brenner, Eds. New York: Springer, 2004, ch. 13.

[9] C. Gimkiewicz, D. Hagedorn, J. Jahns, E. B. Kley, and F. Thoma, "Fabrication of microprisms for planar-optical interconnections using analog gray-scale lithography with high energy beam sensitive glass," Appl. Opt., vol. 38, pp. 2986-2990, 1999.

[10] W. Däschner, P. Long, R. Stein, C. Wu, and S. H. Lee, "Cost-effective mass fabrication of multilevel diffractive optical elements by use of a single optical exposure with a gray-scale mask on high-energy beamsensitive glass," Appl. Opt., vol. 36, pp. 4675-4680, 1997.

[11] E.-B. Kley, "Continuous profile writing by electron and optical lithography," Microelectron. Eng., vol. 34, pp. 261-298, 1997.

[12] E.-B. Kley, L.-C. Wittig, M. Cumme, U. D. Zeitner, and P. Dannberg, "Fabrication and properties of refractive micro optical beam shaping elements," Proc. SPIE, vol. 3879, pp. 20-31, 1999.

[13] S. Kopetz, E. Rabe, W. J. Kang, and A. Neyer, "Polysiloxane optical waveguide layer integrated in printed circuit board," Electron. Lett., vol. 40, pp. 668-669, 2004.
[14] A. L. Glebov, J. Roman, M. G. Lee, and K. Yokouchi, "Optical interconnect modules with fully integrated reflector mirrors," IEEE Photon. Technol. Lett., vol. 17, pp. 1540-1542, 2005

[15] P. Dannberg, G. Mann, L. Wagner, and A. Bräuer, "Polymer UV-moulding for micro-optical systems and opto-electronic integration," Proc. SPIE, vol. 4179, pp. 137-145, 2000

Richard Heming received the Diplom (FH) degree from the University of Applied Sciences, Duesseldorf, Germany, in 1995 and the Diplom II degree from the University of Hagen, Germany, in 2005, both in electrical engineering, where he is currently pursuing the doctoral degree.

Currently he is an R\&D Engineer with the Institute of Analytical Sciences, Dortmund, Germany. His research interests are in the fields of microoptics, optical spectroscopy, and electronic circuit design.

Lars-Christian Wittig received the Diploma and Ph.D. degrees in physics from the University of Jena, Germany, in 1999 and 2005, respectively.

Currently, he is with the Optical Design Department, Carl Zeiss, Jena. His research interests include grayscale lithography using variable dose writing and the design and fabrication of microoptical elements, especially for optical beam shaping.

Peter Dannberg received the Diplom and doctoral degrees in physics from the University of Jena, Germany, in 1983 and 1987, respectively.

Since 1993, he has been with the Fraunhofer Institute of Applied Optics and Precision Engineering, Jena, Germany, where he is currently Head of the Microoptics Technology Group. His interests include microoptical elements and systems as well as polymer optical materials and microstructuring.

Jürgen Jahns received the Diplom and doctoral degrees in physics from the University of Erlangen, Germany, in 1978 and 1982, respectively.

From 1983 to 1986, he was with Siemens AG, Germany. From 1986 to 1994, he was with AT\&T Bell Laboratories, Holmdel, NJ. Since 1994, he has been a Professor of optical information technology at the University of Hagen, Germany.

Ernst-Bernhard Kley is Head of the Microstructure Technology and Microoptics Group, Institute of Applied Physics, University of Jena, Germany.

Matthias Gruber received the Diplom degree in physics from the University of Erlangen, Germany, in 1991 and the doctoral degree in electrical engineering from the University of Hagen, Germany, in 2003.

$\mathrm{He}$ is currently an Assistant Professor of optical microsystems at the University of Hagen. 